\title{
Estrategias educativas prevención de embarazo en adolescentes. Unidad Educativa UPSE
}

\section{Educational strategies for teenage pregnancy prevention UPSE Educational Unit}

\author{
Aida García $^{1 *}$, Carlos Saavedra $^{1}$, Nancy Domínguez ${ }^{1}$, Isoled Herrera ${ }^{1}$ y Adonis Hernández $^{1}$ \\ ${ }^{1}$ Universidad Estatal Península de Santa Elena \\ *aidita66hildifer@gmail.com
}

DOI: https://doi.org/10.26871/killkana_salud.v3i3.490

\begin{abstract}
Resumen
Introduccion: El embarazo en adolescentes es un problema social y de salud pública a nivel mundial, por el incremento de embarazos que se presentan en jóvenes que no son adultas, no tienen la suficiente madurez y preparación para enfrentar la llegada de un nuevo ser y desde el punto de vista bio-psico-social todavía no están aptas para la maternidad. Objetivos: implementar estrategias educativas para elevar el nivel de conocimiento en los adolescentes de la Unidad Educativa UPSE, en Santa Elena, sobre la prevención del embarazo a temprana edad, e identificar los factores causales que influyen en el embarazo en adolescentes. Materiales y Métodos: La investigación estuvo enmarcada en un estudio descriptivo, cualicuantitativo y transversal. La población muestra de estudio estuvo representada por 375 adolescentes con edades de 14 a 19 años que representaron el $53 \%$ de la población total, entre ellas 4 embarazadas, 3 son madre y 368 sin gestar. Para la recolección de datos se aplicó la encuesta como instrumento. Resultados Se obtuvo como resultados que el $57 \%$ iniciaron precozmente representando el grupo etario de 15 a 16 años seguida el grupo de 12 a 13 años con un 25,3\% y el 79,2\% conocen sobre los métodos anticonceptivos, mientras que el 20,8\% no tienen conocimiento. Conclusiones La importancia de esta investigación, en el ámbito de enfermería, radica en la necesidad de generar acciones educativas, tanto para la población adolescente como la adulta, que no solo beneficiará a los adolescentes sino a las familias, a las comunidades, al sector educativo de salud y a los profesionales de la salud. De igual manera el sistema de salud ya que contribuyendo a disminuir los embarazos en adolescentes se disminuirán también las muertes maternas ya que constituye una de las primeras causas de muertes.
\end{abstract}

Palabras clave: Adolescentes, Embarazo, Factor de riesgo, Métodos Anticonceptivos, Prevención.

\begin{abstract}
Introduction: Teenage pregnancy is a worldwide social and public health problem, especially because of the increase in the number of pregnancies occurring in young girls who are not mature and therefore, do not have sufficient maturity and preparation for facing the arrival of a new human being, since from the bio-psycho-social point of view they are not yet ready for maternity. Objectives: To implement educational strategies for the adolescents at the UPSE Educational Unit in Santa Elena to raise their knowledge level about early pregnancy prevention, and to identify the causal factors influencing teenage pregnancy. Materials and Methods: The research was carried out within a descriptive, qualitative, quantitative and cross-sectional study framework. The study sample population consisted of 375 adolescents aged 14 to 19 years who represented 53\% of the total population. It included 4 pregnant girls, 3 of them were already mothers and 368 were not pregnant. The survey was the tool used for data collection. Results: Results show that $57 \%$ of the participants started sexual intercourse early in life, they represented the 15 to 16 years age group, followed by the 12 to 13 years age group, with 25.3\%; 79.2\% knew about contraceptive methods, while 20.8\% lacked this knowledge. Conclusions: The importance of this research, in the nursing field, resides in the need for generating educational actions, both for the adolescent and the adult population; which will not only benefit adolescents but families, communities, the health education sector, health professionals, and the health system, since, by contributing to the reduction of teenage pregnancies, the number of maternal deaths will also decrease as teenage pregnancy constitutes one of the main death causes.
\end{abstract}

Key words: Adolescents, Pregnancy, Risk factor, Contraceptive methods, Prevention. 


\section{Introducción}

La Organización Mundial de la Salud(OMS) define la adolescencia como el periodo de crecimiento y desarrollo humano que se produce después de la niñez y antes de la edad adulta; entre las etapas de 10 y los 19 años, se distinguen dos tramos: la adolescencia precoz de (1014 años) y la tardía de (15-19 años). Alguna/os autores identifican tres etapas de acuerdo a los cambios físicos y evolutivos: adolescencia temprana de (10 a 13 años), media (14 a 16 años) y tardía ()17 a 19 años). Se trata de una de las etapas de transición más importantes en la vida del ser humano, caracterizándose por un ritmo acelerado de crecimiento y de cambios. Esta fase de crecimiento y desarrollo viene condicionada por diversos procesos biológicos. El comienzo de la pubertad marca el pasaje de la niñez a la adolescencia.

La adolescencia es un período de grandes cambios y transformaciones: el cuerpo del niño o niña comienza a madurar, a convertirse en adulto, su mente se llena de nuevas ideas y pensamientos, aparecen nuevos gustos e intereses. Es la época del deseo de independencia familiar, del inconformismo y de las teorías que pretenden cambiar el mundo. En este momento nacen las grandes amistades y los primeros amores que siempre dejan huella, está asociado con un mayor índice de morbilidad y mortalidad, tanto para la madre como para el bebé. El organismo de la adolescente tiene unas necesidades para su propio desarrollo en las que puede interferir la gestación, con los consiguientes riesgos de malnutrición o retraso en el desarrollo óseo, entre otros.

En este sentido, de acuerdo con la Organización de Naciones Unidas (ONU) (2012), a nivel mundial las probabilidades de que jóvenes de 15 a 19 años mueran durante el embarazo o el parto duplican las de las mujeres con más de veinte años. Ese riesgo es cinco veces mayor en el caso de las niñas menores de 15 años. Igualmente, la OMS (2010), estima que el riesgo de morir a consecuencia de problemas relacionados con el embarazo es el doble para las mujeres entre 15 a 19 años, que para las mujeres de 20 a 24 años y para las de 10 a 14 años (Adolescencia Temprana), las tasas de mortalidad materna pueden llegar a superar hasta cinco veces la de las mujeres embarazadas que tienen entre 20 a 24 años.

La mayoría de los países de Centro América, entre ellos: Nicaragua, Honduras, Guatemala, Venezuela, República Dominicana y de América del Sur: Ecuador, registran los índices más altos de jóvenes de 15 y 19 años que son madres. Ecuador ocupa el primer lugar en la región andina, y el segundo en América Latina superado por Venezuela, respecto a embarazos en adolescentes. El Instituto Nacional de Estadística y Censos(INEC) señala que, en los últimos 10 años, el incremento de partos de adolescentes entre $10 \mathrm{y}$ 14 años fue del $78 \%$ y en adolescentes entre 15 a 19 años del $11 \%$. También muestra que en la provincia de Santa Elena el total de madres adolescentes de 12 a 19 años es de 3.090 .
La atención a la salud sexual y reproductiva de las y los adolescentes constituye una prioridad de salud pública a nivel internacional, nacional y regional no solo por el porcentaje de la población que este grupo etario representa, sino por la trascendencia en la toma de decisiones y comportamientos que se dan en este ciclo de vida y que pueden afectar su futuro. El embarazo en la adolescencia no solo representa un problema de salud, también tiene una repercusión en el área social y económica, dado que implica menores oportunidades educativas o el abandono total de los estudios por parte de la madre adolescente, lo que incide en el incremento de la exclusión y de las desigualdades de género, coadyuvando en el fortalecimiento del círculo de la pobreza.

De igual forma, debido a que el embarazo en la adolescencia no es planificado y es considerado como un problema de salud pública por la falta de prevención, información sobre los métodos anticonceptivos y su concepción, esto no es suficiente para explicar el por qué las adolescentes quedan embarazadas, ya que son muchos los factores que pueden influir en este hecho. Sin embargo, es necesario tener presente que constituye un riesgo médico muy alto, que lleva consigo dificultades biológicas, físicas, socioeconómicas y emocionales para la madre y su hijo. Es una etapa que puede determinar el sentido de la vida y el bienestar futuro de la adolescente.

Por esta razón, es necesario ofrecer una atención especial centrada en la prevención del embarazo a temprana edad, los embarazos repetidos y la lucha contra las enfermedades de transmisión sexual, incluida el VIH/SIDA. El presente estudio tiene como finalidad la ejecución de un plan de intervención educativa para prevenir el embarazo en adolescentes de la Unidad Educativa UPSE La Libertad, Santa Elena. En el cual debe haber una disposición a trabajar en pro de dar ayuda para contribuir en la ampliación de estrategias educativas dirigidos a todos los adolescentes en todas las instituciones de Salud de la provincia de Santa Elena asi como a las adolescentes que ya son madres precozmente para evitar un segundo embarazo y disminuir las complicaciones que se derivan de ellos, y por ende, las incidencias de embarazo precoz.

En julio de 2018 se presentó la Política Intersectorial de Prevención del Embarazo en Niñas y Adolescentes 20182025. El mismo tiene como objetivo contribuir a que las y los adolescentes accedan de manera universal a información, educación y educación integral de la sexualidad, servicios de salud sexual y salud reproductiva, para la toma de decisiones libres, responsables y saludables sobre su sexualidad y reproducción, a través del ejercicio pleno de los derechos sexuales y derechos reproductivos para una vida libre de violencia.

Situación demográfica y económica: la adolescencia (10 a 19 años) y juventud (20 a 24 años) en el Ecuador constituyen el $31 \%$ de la población. Los costos económicos y sociales a futuro serán considerables si este grupo no llega a la edad adulta con buena salud, educación y la capacidad 
de responder a las necesidades personales y colectivas, y la posibilidad de participar en la sociedad de forma que puedan impulsar el desarrollo del país. Adolescentes sanos y competentes que ingresan a la fuerza laboral pueden aumentar la productividad económica de un país.

Este bono demográfico termina en Ecuador en el año 2030, por lo que es en este momento cuando se debe aprovechar tanto para promover el crecimiento saludable, la adquisición de competencias y el desarrollo integral como para prevenir las consecuencias de potenciales conductas de riesgo. En la adolescencia, se instauran prácticas y estilos de vida que, si son positivos, garantizarán la calidad de vida en la adultez; en caso contrario, se afecta dicha calidad. (Calderón Morales I C, 2014 ) En esta etapa se definen los proyectos de vida, los cuales se convierten en factores protectores primordiales.

En el Ecuador como resultante del desarrollo social y las políticas de salud, los adolescentes disfrutan de derechos a educación sexual, planificación familiar y acceso universal a servicios, incluida asesoría, información y comunicación en reproducción humana y sexualidad. Sin embargo es evidente que un número considerable de individuos carecen aún de comportamientos sexuales y reproductivos responsables, en lo que juega un papel importante la familia, el grupo de pares y la sociedad. En la actualidad se implementan nuevas medidas y estrategias para la prevención de este problema de salud. De esta forma se plantean las siguientes interrogantes:

¿Cómo elevar el nivel de conocimientos de las (os) adolescentes en la Unidad Educativa UPSE, sobre el embarazo a temprana edad?

¿Cuáles son los factores causales que inciden en el incremento de embarazo en adolescente en los estudiantes de la carrera de enfermería, Universidad Península de Santa Elena, La Libertad, durante el primer semestre del año 2018 ?

Este trabajo de investigación es de suma importancia ya que servirá como: Guía para los estudiantes de Salud, referencia bibliografía, aporte para la Ciencia de la Salud, iniciativa para nuevas investigaciones sobre la adolescencia, material de información para las/los Lic. en Enfermería y de esta forma concientizar a todos los profesionales de la salud sobre la problemática que conlleva el embarazo en la adolescencia; e incrementar la educación sexual y salud reproductiva mediante estrategias a través de talleres, realizar charlas educativas respecto a los adolescentes sobre planificación familiar, embarazo y la anticoncepción en los escenarios educativos.

También se beneficiarán los estudiantes de licenciatura de enfermería ya que se formarán mejores profesionales capaces de crear estrategias educativas que disminuyan las problemáticas de salud. A pesar de la gran importancia y las estrategias creada por el Ministerio de Salud Pública(MSP), aún existen desconocimientos en la forma que pueden prevenirse los embarazos en adolescentes, por lo que se debe continuar trabajando en la educación para la salud en todos los escenarios con la participación de los profesionales para mejorar la calidad de vida del individuo, la familia y la comunidad, en consideración los aspectos físicos, psicológicos y sociales

\section{Marco Teórico}

\section{Antecedentes investigativos}

Algunos trabajos que se realizaron anterior y que abordan la problemática del embarazo en adolescentes son los siguientes:

Organismo Andino de Salud (2010): En un estudio estima que cerca de siete millones de mujeres adolescentes de entre 15 y 19 años que viven en la subregión andina, al menos un millón ya son madres o están embarazadas. Este fenómeno causa, además, pérdida de vidas con el consiguiente costo de capital humano, puesto que el riesgo de morir antes, durante o después del parto es cuatro veces mayor entre las menores de 16 años que en las mayores de 20, al mencionar un estudio publicado en la revista científica American Journal of Obstetrics and Gynecology.

(Osotimehin, (2010))Explica en un informe los riesgos que generan los embarazos para la salud de las niñas y adolescentes. Cerca de 70.000 adolescentes mueren cada año en el mundo por causas relacionadas con la gestación y el nacimiento. El informe aporta testimonios de chicas que han sido madres, como Swinton, una zimbabuense que quedó embarazada a los 15 años.

(Ysazzi, 2010) Consejera en salud sexual y reproductiva asevera: "Es lamentable la desinformación y los mitos y tabúes que se dan en la familia y la escuela y que han abocado a la mujer venezolana a abortos y enfermedades de transmisión sexual". Las adolescentes no suelen usar métodos anticonceptivos en sus primeras relaciones sexuales y, a pesar de que nueve de cada diez los conoce, solo una de cada diez los usa, según datos de la Encuesta Nacional de Población y Familia.

(Mora, 2009) Director adjunto del Fondo de Población de Naciones Unidas para América Latina y el Caribe, en una entrevista con EFE en un foro que analiza los resultados del Plan Andino de Prevención del Embarazo Adolescente, afirmó: "Muchas madres adolescentes abandonan la escuela y al hacerlo interrumpen su educación, por lo que muchas de ellas tienen que acceder a empleos precarios". Agregó que en esos casos, "se produce un ciclo intergeneracional de la pobreza, porque ocurre con mucha frecuencia que los hijos e hijas de esas madres tempranas a su vez también tienen embarazos tempranos y truncan o limitan su proyecto de vida".(p.25).

\section{Teorías existentes sobre el tema familia como paciente y estructura familiar}

Al generalizar la teoría de Duvall (2009), se puede considerar a la familia como un grupo pequeño que cambia y evoluciona en el tiempo. La vida familiar está dividida en etapas sucesivas que comienza con la unión de la pareja y termina con la muerte de uno de sus miembros, o con la 
separación física de la pareja. En cada una de esas etapas existen deberes de la familia que son fundamentales para la preservación y desarrollo armónico de la misma.

De acuerdo con Duvall (2009) todas las familias tienen estos deberes básicos mientras existan. Cada una realiza estas funciones a su manera, es decir, bajo sus propias normas únicas. La enfermera obtiene datos para ratificar la forma en que la familia está cumpliendo cada uno de estos deberes, en particular los relacionados el mantenimiento y prevención de la salud.

La adolescencia es el periodo de la vida en el cual el individuo adquiere la capacidad reproductiva. Es una etapa muy importante en el curso de la vida en la cual se producen cambios biológicos (crecimiento, caracteres sexuales secundarios y capacidad de reproducción), psicológicos (pensamiento abstracto) y sociales (pertenencia grupal) transitan los patrones psicológicos de la niñez a la adultez, consolida la independencia socio-económica y fija sus límites aproximados entre los 10 y 20 años. En este período, se profundiza el proceso de construcción de la identidad, la autonomía, la sexualidad, la vocación y los proyectos de vida. A medida que los adolescentes experimentan estos cambios, pueden probar muchos comportamientos de riesgo entre los que se encuentran la actividad sexual.

Según la Organización Mundial de la Salud (OMS), se estima que el $70 \%$ de las muertes prematuras en el adulto se deben a conductas iniciadas en la adolescencia que representa el momento clave para la incorporación de hábitos necesarios para adquirir buena calidad de vida. Invertir en la adolescencia acelera la lucha contra la pobreza, las disparidades socioeconómicas, la discriminación por razones de género, y garantiza el cumplimiento de los derechos.

De acuerdo al Censo de Población y Vivienda de 2010 del INEC, el 20,5\% de la población es adolescente (2 958 879); el 10,6\% son adolescentes tempranos de 10 a 14 años (1 539 342) y, el 9,8 son adolescentes tardíos entre 15 a 19 años (1 419 537). El $49 \%$ mujeres y $51 \%$ hombres; $60,6 \%$ reside en áreas urbanas y 39,4\% en áreas rurales. El 68,6\% de jóvenes urbanos (antes 40,1 \%) han terminado la educación secundaria, comparando con el 36,1\% (antes $14,6 \%$ ) en el área rural. Las mayores brechas educativas se observan en adolescentes auto identificados como indígenas y montubios.

Cuatro de cada 10 jóvenes de áreas urbanas y ocho de cada 10 de áreas rurales son pobres de acuerdo a necesidades básicas insatisfechas, cerca de la mitad de ellos viven en condiciones de extrema pobreza, y dos de cada 10 adolescentes entre 15 a 18 años ha migrado al exterior. En el grupo de adolescentes tardíos de 15-19 años, la carga de morbilidad está ligada a síndromes y patologías que afectan la salud sexual y salud reproductiva, otras que demandan resoluciones quirúrgicas y relacionadas con la violencia. La mortalidad se explica, en de la mitad de los casos, por causas asociadas a accidentes de tránsito, a la violencia, lesiones autoinfligidas y ahogamiento.

Las causas más comunes de muerte son el suicidio en las adolescentes (13\% de todas las muertes) y las lesiones de tráfico para los muchachos (19\%) (CNII 2014). Los accidentes de tráfico fueron la segunda causa más común de muerte para las adolescentes (8\%). Para los adolescentes, la violencia fue la segunda causa más común (12\%), y el suicidio del tercero $(7 \%)$. La violencia, incluida la violencia infligida por la pareja, es una causa importante de morbilidad y mortalidad para ambos sexos. El principal problema en materia de salud sexual y reproductiva es la alta tasa de embarazos de adolescentes. Estas altas tasas se extienden a adolescentes menores de 15 años de edad (2014) (CNII).

El MSP promueve la implementación de servicios diferenciados para la atención integral de adolescentes en unidades de primer nivel y en hospitales básicos a partir del desarrollo del paquete normativo para la atención integral de adolescentes (norma, protocolo y caja de herramientas), de sensibilización y capacitación con los enfoques de género, intercultural, intergeneracional, de diversidad, derechos, en especial, los derechos sexuales y reproductivos, al personal multidisciplinario de los establecimientos de salud.

En el año 2010, se genera la Estrategia Intersectorial de Planificación Familiar y Prevención del Embarazo en la Adolescencia (ENIPLA). Estos antecedentes han sido parte del proceso para la institucionalización de la atención integral para adolescentes en el primer nivel, en el marco de conceptualización de la atención bio-psico-social de calidad, con énfasis en la prevención y promoción, que brinda atención a cada persona de una manera integral e impulsa el desarrollo humano.

El Ministerio de Salud Pública, en su calidad de Autoridad Sanitaria Nacional, emitió en marzo del 2017 el "Plan Nacional de Salud Sexual y Salud Reproductiva 2017 - 2021”, actual política pública en salud sexual y salud reproductiva que busca fortalecer la rectoría, redefinir el enfoque en la provisión integral de servicios de salud, reducir las brechas en la provisión servicios de salud sexual y salud reproductiva integrales.

Este Plan tiene como marco de actuación los enfoques en salud propuestos en el Modelo de Atención Integral de Salud-Familiar, Comunitario e Intercultural (MAIS-FCI): derechos humanos, igualdad, género, interculturalidad, intergeneracionalidad, inclusión social, promoción, participación y ciudadanía. También considera la problemática actual relacionada con la vivencia de la sexualidad, los derechos sexuales y derechos reproductivos, en reconocimiento de los derechos humanos fundamentales que deben ser garantizados y efectivizados por parte del Estado, como lo mencionan la Constitución y los instrumentos internacionales. 


\section{Metodología}

El presente estudio es cuantitativo- cualitativo, descriptivo, de corte transversal, ya que se toma un grupo etáreo de edades entre 12 y 19 años, se identifica la naturaleza profunda de las realidades, su sistema de relaciones y su estructura dinámica basándose en la observación de los hechos sin explicarlos. Se realiza un corte en el tiempo. En este caso, es el $2^{\circ}$ semestre del año 2018.

\section{Universo y muestra}

Área de estudio: Unidad Educativa UPSE, La Libertad, Santa Elena, 2018. El universo de estudio es de 707 adolescentes del sexo femenino y masculino entre 14 y 19 años. La muestra fue de 375 adolescentes que aceptaron participar en el estudio, que representa el 53,04\% del total de la población adolescente del universo. Esta población de estudio está conformada por los estudiantes de primero y segundo de bachillerato de la Unidad Educativa UPSE. La técnicas de instrumento utilizada fue a través de encuestas anónimas, a adolescentes entre 14 y 19 años de los que contendrán preguntas directas. Esta técnica permitió recoger información de primera mano por medio de opiniones.

\section{Resultados}

El siguiente estudio pretende evaluar el nivel de conocimiento que poseen los estudiantes adolescentes con temas relacionados a salud sexual y reproductiva. De los 375 estudiantes, el 79,2\% conocen sobre los métodos anticonceptivos y $20,8 \%$ no tienen conocimiento sobre este tema.

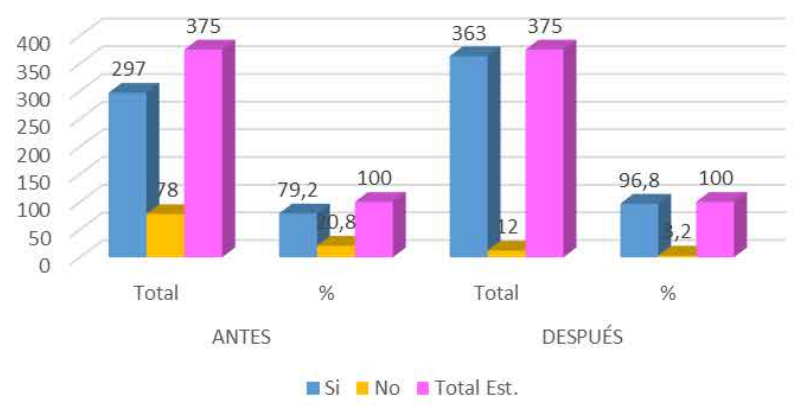

Fig. 1. Distribución conocimientos sobre métodos anticonceptivos Fuente: Encuesta aplicada a los adolescentes UE. UPSE

Antes de la intervención, el 79, $2 \%$ de los adolescentes estudiados tenían conocimientos sobre los métodos anticonceptivos, y el 20,8\% tenían conocimientos inadecuados. Luego de la intervención, se logró que el 96,8\% de los estudiantes tuvieran conocimientos adecuados con los talleres realizados y la participación de los estudiantes del quinto semestre que participan proyecto de vinculación.

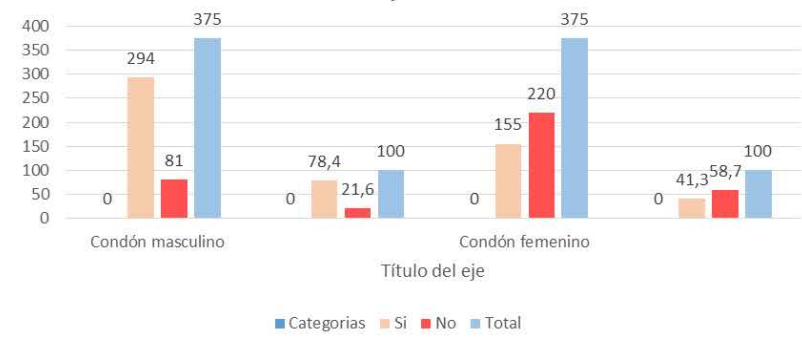

Fig. 2. Distribución conocimientos sobre uso del condón masculino y femenino

Fuente: Encuesta aplicada a los adolescentes UE. UPSE

El 78,4\% de los adolescentes conocían el condón masculino y el $21,6 \%$ no lo conocían, mientras que relacionado al condón femenino el 58,7 \% no lo conocían y el 41,3\% si lo conocían

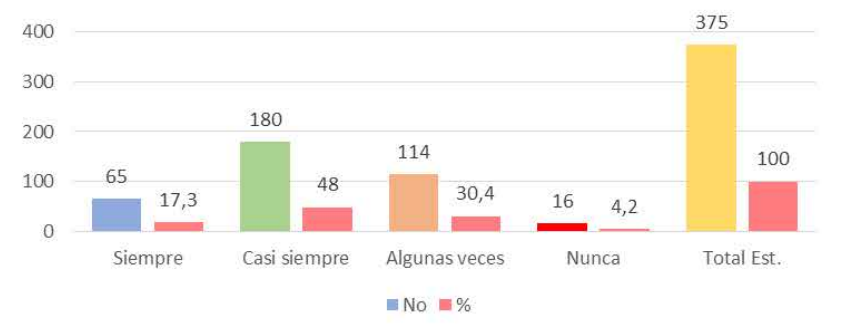

Fig. 3. Distribución el uso adecuado de los métodos anticonceptivos previene el embarazo en adolescentes y las enfermedades de trasmisión sexual

Fuente: Encuesta aplicada a los adolescentes UE. UPSE

El $48 \%$ de los adolescentes refieren que casi siempre el uso adecuado del condón previene los embarazos en adolescentes y las enfermedades de transmisión sexual y el $17 \%$ refieren que siempre, identificándose el poco conocimiento que tienen salud sexual y reproductivo así como de la planificación familiar.

Otros autores refieren que es frecuente que las adolescentes ignoren los riesgos y daños que pueden significar las relaciones sexuales desordenadas, entre ellos el embarazo.

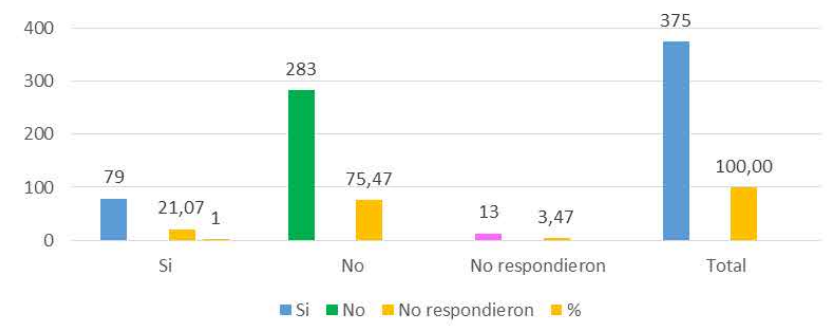

Fig. 4. Distribución si ha tenido relación sexual

Fuente: Encuesta aplicada a los adolescentes UE. UPSE

De los 375 estudiantes que representan el $100 \%$ de la muestra el 21,07\% (hombres y mujeres) iniciaron sus relaciones sexuales, el 75,47\% no inician relaciones sexuales, 
con lo que se obtiene una similud los resultados con el trabajo presentado de de Guillén Terán, A. M. (2015).

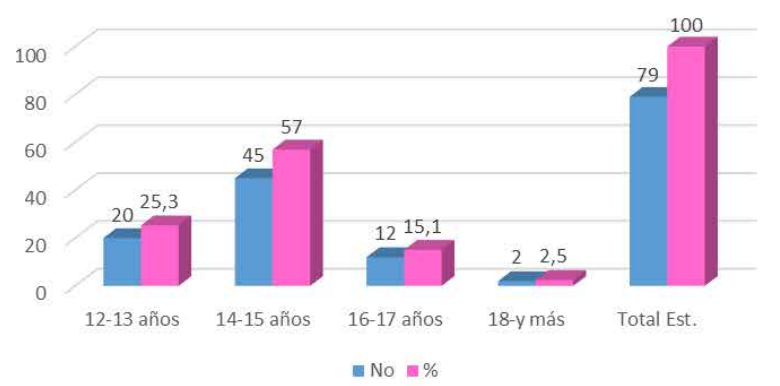

Fig. 5. Distribución edad de las relaciones sexuales

Fuente: Encuesta aplicada a los adolescentes UE. UPSE

El $21 \%$ de los adolescentes tuvieron sus relaciones sexuales antes de los 18 años, con el predominio de los grupos de edades de 14 a 15 representando el $57 \%$ seguido los grupos de 12 a 13 años. Esto demuestra que existe un ascenso de inicio precoz de las relaciones sexuales en coincidencia con otros autores como es el caso Correoso, M. V., Dandicourt Thomas, C., \& Soria, Y. M. (2012).

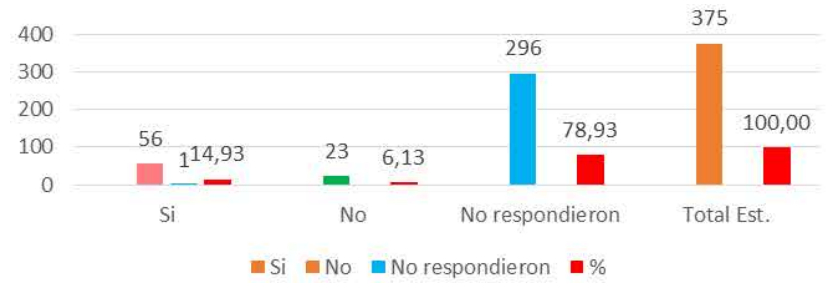

Fig. 6. Distribución de métodos anticonceptivos utilizados en la primera relación sexual

Fuente: Encuesta aplicada a los adolescentes UE. UPSE

Relacionado con los métodos anticonceptivos utilizados en las primeras relaciones sexuales el 14,9\% refirió haber usado algunos métodos anticonceptivos, mientras que el $6 \%$ refirió no haber usado ningún método de protección. El método más usado fue el preservativo con un 64,6 por ciento.

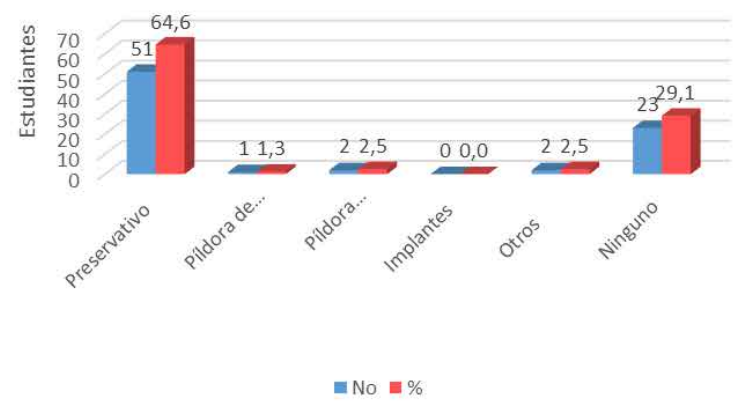

Fig. 7. Distribución tipos de métodos anticonceptivos utilizados Fuente: Encuesta aplicada a los adolescentes UE. UPSE
El método anticonceptivo más utilizado por los adolescentes fue el preservativo, lo que representa el 64,6\% mientras que el 29,1 refirió no usar ningún método anticonceptiv;, lo que no demuestra la poca información del uso de los mismos tanto para la prevención del embarazo como para la prevención de embarazo en adolescentes, en concordancia con el trabajo realizado por Guillén Terán, A. M.. ${ }^{1}$ Para el $44 \%$ de los encuestados el método de mas uso fue el preservativo.

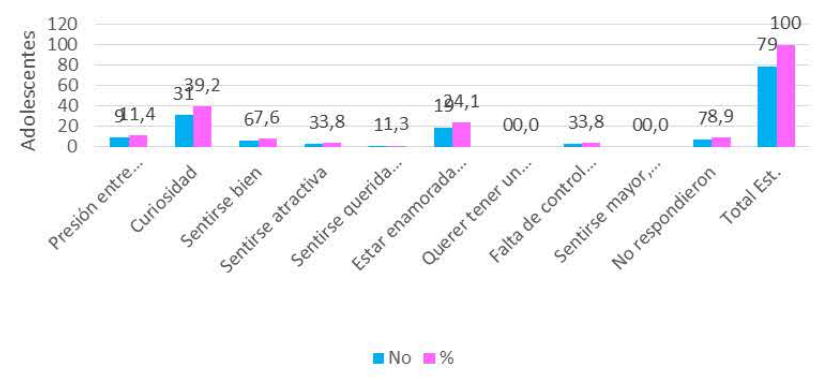

Fig. 8. Distribución que motivo tener relaciones sexuales Fuente: Encuesta aplicada a los adolescentes UE. UPSE

De acuerdo a los motivos por el inicio precoz de las relaciones sexuales, en este estudio los adolescentes expusieron que fue por curiosidad representando el 39,2 \% seguida por estar enamorados.

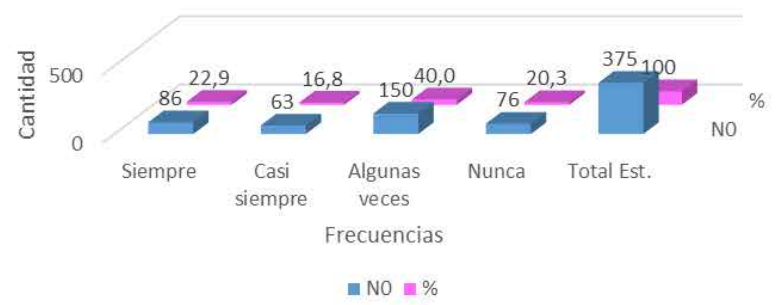

Fig. 9. Distribución frecuencia que los familiares hablan sobre sexualidad

Fuente: Encuesta aplicada a los adolescentes UE. UPSE

El $40 \%$ de los adolescentes aludieron que algunas veces sus familiares le hablaban sobre la sexualidad y el 22,9 expresaron que siempre mantienen comunicación sobre la sexualidad. El 54,7\% de los adolescentes refirieron que en la escuela le hablan sobre temas relacionados a la sexualidad, y se pudo evidenciar que el $55,7 \%$ no tenían los conocimientos suficientes sobre sexualidad. Para el 44,3\% se constató que si tenían conocimientos sobre la sexualidad. 


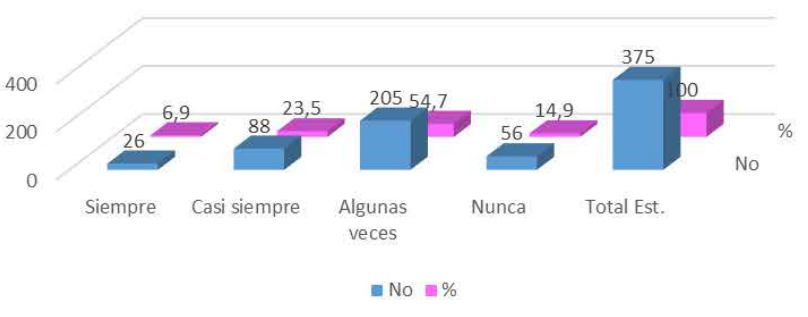

Fig. 10. Distribución frecuencia que en la escuela hablan sobre sexualidad

Fuente: Encuesta aplicada a los adolescentes UE. UPSE

Del $100 \%$ de los estudiantes encuestados relacionados con la educación sobre sexualidad el $6,9 \%$ refieren que siempre le hablan del tema, mientras que el 54,7\% algunas veces y el $14,9 \%$ nunca han recibido información. Esta situación evidencia que no existe establecido en la unidad educativa una asignatura donde los estudiantes puedan adquirir esos conocimientos.

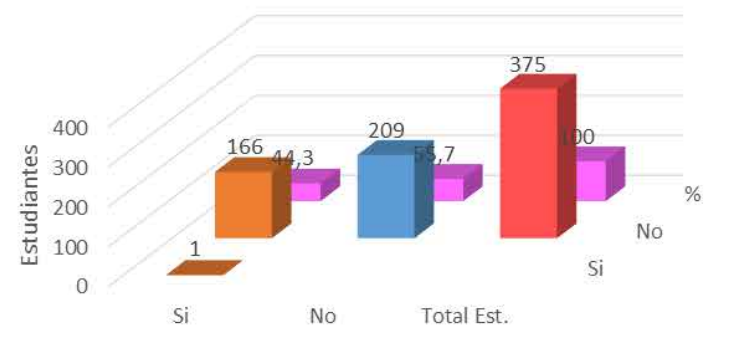

a $\mathrm{Si}=\mathrm{No}=\%$

Fig. 11. Distribución sabes lo suficiente de sexualidad

Fuente: Encuesta aplicada a los adolescentes UE. UPSE

El $55,7 \%$ de los estudiantes refieren no tener suficiente conocimiento sobre la sexualidad, y el $44 \%$ manifestó tener algún conocimiento.

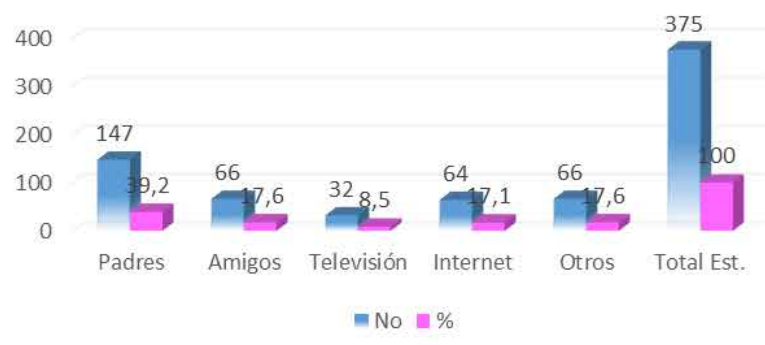

Fig. 12. Distribución medios de comunicación recibió la información

Fuente: Encuesta aplicada a los adolescentes UE. UPSE

Por los medios de comunicación que los adolescentes recibieron la mayor información fue a través de los padres con el $39,2 \%$, seguidas por los amigos con el $17,6 \%$ en concordancia con Correoso, M. V., Dandicourt Thomas, C., \& Soria, Y. M. ${ }^{2}$ que en su trabajo los adolescentes obtuvieron mayor información a través de los padres.

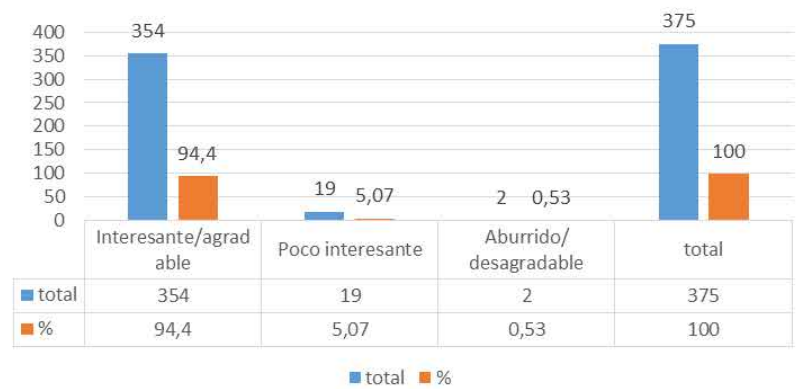

Fig. 13. Satisfación sobre evaluación de los talleres realizados

Fuente: Encuesta aplicada a los adolescentes UE. UPSE

El 94,4\% de los adolescentes evaluaron de interesante los talleres realizados para incrementar los conocimientos sobre salud sexual y reproductiva por los estudiantes del quinto semestre de la carrera de enfermería, el 5,07\% poco interesante y $0,53 \%$ aburrido.

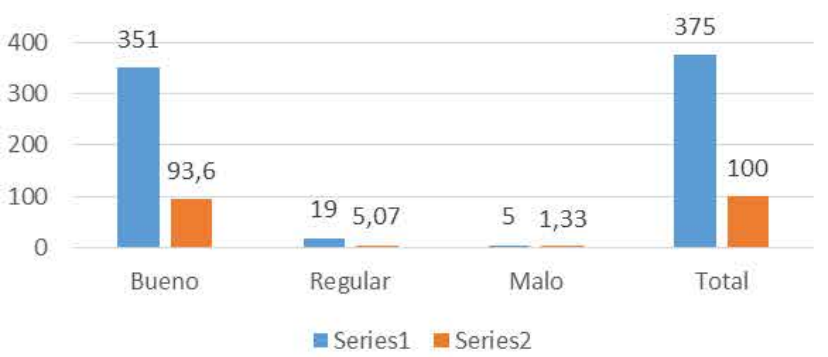

Fig. 14. Evaluación del desarrollo de los talleres

Fuente: Encuesta aplicada a los adolescentes UE. UPSE

El 93,6\% de los estudiantes estudiados evaluaron de bueno los talleres desarrollados con temas de salud sexual y reproductivos, mientras que el 5,07\% evaluaron de regular y el $1,33 \%$ de malo ya que no fueron temas de su interés.

\section{Conclusiones}

A pesar de los esfuerzos de las estrategias y políticas intersectoriales por el Ministerio de salud Pública (MSP) para mejorar los aspectos relacionados con salud sexual y reproductiva en la adolescencia, aún se mantiene vulnerable. El embarazo en la adolescencia continúa siendo un problema de salud pública, por lo que sería indispensable profundizar los programas de información, que si bien existen, no son suficientes. De ahí la necesidad de implementar actividades dirigidas a este grupo vulnerable en cuanto a la sensibilización, aumentar el nivel de conocimiento y responsabilidad con la participación directa de los adolescentes y la familia, ya que la falta de educación sexual como parte integral de la educación e inadecuado manejo de información sobre sexualidad proveniente de los medios no permite disminuirlo. De igual manera predomina en las familias falta de comunicación relacionado a temas de sexualidad, y arraigados patrones culturales que reproducen mitos y tabúes respecto a sexualidad. 
Los adolescentes cada día inician sus relaciones sexuales cada vez más precoz por falta de conocimientos y de información, ignorando las consecuencias y complicaciones que genera el embarazo en esta etapa. El porcentaje de adolescentes que tuvo su primera relación antes de los 18 años ha ido en ascenso predominando los grupos etarios de 14-15 años y de 13 a 14 años. Los motivos del inicio de las relaciones sexuales en adolescentes fue motivado en la mayor parte por curiosidad seguida por el enamoramiento.

Con todo lo anterior expuesto se evidencia que el gobierno ecuatoriano a pesar de las políticas y estrategias diseñadas para la prevención de embarazo en adolescentes no han sido suficientes y no se ha cumplido en su totalidad. Como se evidencia en el caso de la falta de información, la comunicación tanto con la familia como en el entorno educativo, pues los adolescentes carecen de estos conocimientos, lo que no favorece lo solicitado por el Estado y el MSP. Como se ha observado, la población investigada acepta lo formulado y planificado de acuerdo a las actividades propuestas en el proyecto ya que se observa el interés por adquirir todas las informaciones relacionadas con temas de salud Sexual y reproductiva a través de los talleres desarrollados en la institución como beneficiarios y la preparación de promotores de salud.

\section{Recomendaciones}

Incrementar programas de información y capacitación dirigidos a empoderar a los adolescentes para que asuman control sobre su cuerpo y decisiones.

Mejorar la comunicación sobre sexualidad con personas cercanas, como la familia y profesores.

Posponer las relaciones sexuales a edades más avanzadas, si ya inicio su vida sexual, hay opciones como protegerse siempre usando preservativos u otro método anticonceptivo.

Continuar con la realización de talleres educativos y formación de promotores de salud para garantizar la continuidad fortaleciendo las actividades de promoción y prevención en salud sexual y reproductiva para evitar y disminuir los embarazos a temprana edad.

\section{Fuente de Financiamiento}

Este estudio es autofinanciado

\section{Conflicto de Intereses}

No existen conflictos personales, profesionales, financieroso de otro tipo.

\section{Consentimiento Informado}

Los autores cuentan con el consentimiento informado de los paciente para la investigación, la publicación del caso y sus imágenes.

\section{Referencias Bibliográficas}

1. Guillén Terán AM. Nivel de conocimiento y percepción de los y las adolescentes de 14 a 18 años sobre el uso de métodos anticonceptivos frente a la prevención del embarazo no deseado en la Institución Educativa Ludoteca de Septiembre a Diciembre del 2014. 2015;

2. Correoso MV, Dandicourt Thomas C, Soria YM. Prevención del embarazo en adolescentes. Revista Cubana de Enfermería. 2012;28(2):125-135.

Recibido: 15 de diciembre de 2018

Aceptado: 28 de marzo de 2019 